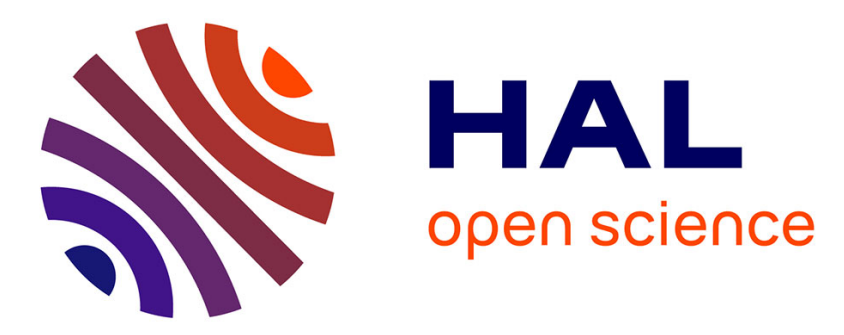

\title{
Spectrally exclusive phase masks for wavefront coding
}

Boher Micke, Julien Jaeck, Patrick Bouchon, Jérôme Primot, Riad Haïdar

\section{To cite this version:}

Boher Micke, Julien Jaeck, Patrick Bouchon, Jérôme Primot, Riad Haïdar. Spectrally exclusive phase masks for wavefront coding. Optics Letters, 2021, 46 (2), pp.436-439. 10.1364/OL.408640 . hal03106866v2

\section{HAL Id: hal-03106866 \\ https://hal.science/hal-03106866v2}

Submitted on 29 Jan 2021

HAL is a multi-disciplinary open access archive for the deposit and dissemination of scientific research documents, whether they are published or not. The documents may come from teaching and research institutions in France or abroad, or from public or private research centers.
L'archive ouverte pluridisciplinaire HAL, est destinée au dépôt et à la diffusion de documents scientifiques de niveau recherche, publiés ou non, émanant des établissements d'enseignement et de recherche français ou étrangers, des laboratoires publics ou privés. 


\title{
Spectrally exclusive phase masks for wavefront coding
}

\author{
Micke Boher, ${ }^{1}$ JULIEN JAeCK, ${ }^{1 *}$ PATRICK BOUChON,${ }^{1}$ Jérôme \\ PRIMOT, ${ }^{1}$ ANd RIAD HAÏDAR ${ }^{1}$ \\ ${ }^{I}$ ONERA The French Aerospace Lab, Chemin de la Hunière, F-91760 Palaiseau, France \\ *julien.jaeck@onera.fr
}

\begin{abstract}
The use of phase masks is necessary for wavefront coding and these are often based on optical path differences.

However, the optical dispersion constrains the resulting device to operate within a restricted spectral bandwidth. Here we propose to remove this constraint thanks to sub-wavelength structuration of the surface. The use of spatial and spectral co-localization properties of these structures allows the production of various spectrally exclusive phase masks on the same area.

In this letter, we demonstrate both theoretically and experimentally a component able to locally and independently modify the Point Spread Function (PSF). The realized sample modifies independently the PSF in two spectral bands of interest.
\end{abstract}

(C) 2020 Optical Society of America under the terms of the OSA Open Access Publishing Agreement

Since the 2000s, a new family of optical devices has emerged, often grouped under the term wavefront coding [1]. In this context, a phase mask is used to transform the point spread function in order to introduce features which can be used to extract information in a scene through image processing functions. Initially intended to extend the depth of field in imagery, this technique allows other applications such as distance measurement [2]. The purpose of this paper is to present a new way of coding a phase mask. Based on metasurfaces created with nano-antennas, those components allow a greater flexibility in phase mask engineering. In particular, they allow to define completely independent point spread functions for separate spectral domains.

Metasurfaces are used to shape the incident wavefront by acting on the amplitude, phase and polarization for several applications such as holography [3] or metalenses [4]. There are different categories of metasurfaces based on the materials used [5-8]. All-dielectric structures usually operate in transmission and use a combination of dielectric materials to locally modify the effective index. They can be used in integrated photonics [9] or to develop polarimetric wavefront sensor [10] for example. In order to work at several wavelengths, as dielectric metasurfaces use an effective index, a tradeoff must be find between efficiency and spectral bandwidth [11,12]. A solution is to create multilayer dielectric metasurfaces which are complex to design [13]. It is also possible to decouple the dispersion response of dielectric metasurface [14] but the scaling to additional optical functions is even more complex. Structures based on the use of metallic dipolar antennas have also been studied [15] and metallic plasmonic antennas can have application in the context of multi-wavelength holography [16]. In both cases, the low efficiency and the high chromatism of these structures limit their use in wavefront coding.

Finally metallic resonant structures allow an improvement of the efficiency at the expense of a reflective device. The Metal-Insulator-Metal (MIM) antenna is a three-layer metasurface with great angular tolerance. The first MIM layer of this device is a continuous gold layer optically opaque which means that we will create a reflective surface, the second layer is an insulator cavity and the third one is a textured gold layer. By shaping this last layer, MIM antennas make it possible to perform different optical functions such as totally absorbing light at different specific wavelengths within a single device [17], converting the incident polarization [18] or even reproducing existing optics like lenses $[19,20]$. 
In this letter, we use rectangular MIM nano-antennas (see figure 1(a)) and propose to leverage their interaction cross-section to combine multiple spectrally exclusive phase masks on the same area. We thus demonstrate both theoretically and experimentally a component able to locally and independently modify the point spread function in two spectral bands of interest.

The MIM nano-antennas behave like Fabry-Perot resonators [21] whose resonant wavelength is mainly determined by the length of the antenna which can be changed to tune the phase delay. In figure 1(b) we represent the phase of the reflected TM-polarized light at normal incidence for two lengths of the metallic antenna as a function of the wavelength in the infrared range. Each array exhibits a near $2 \pi$-phase variation that is due to the cavity resonance occurring below the gold patch. As we can see on this figure, when we increase the length of the rod, the resonant wavelength increases and the phase step shift towards longer wavelengths. The MIM are undercoupled thanks to a tradeoff between the period and the $\mathrm{SiO}_{2}$ thickness so the associated absorption is below $30 \%$ (see supplemental material).

The dimensions on figure 1(b) were chosen to obtain a phase difference of $\pi$ at 5 microns between the two different structures characterized by one length of nano-antenna. The bandwidth, defined by $|\Delta \phi-\pi|<0.3 \pi$, is about $1 \mu \mathrm{m}$ in this case. We note that before $3 \mu \mathrm{m}$ and after $7 \mu \mathrm{m}$ the two antennas exhibit no phase difference. We use the two structures to realize the sample in figure 1(a) consisting in two spatially distinct areas of a periodic pattern of nano-antennas, in each area the length of the antenna will impose the phase. Outside of the bandwidth of interest (here $5 \mu \mathrm{m} \pm 500 \mathrm{~nm}$ ) the device will not show any phase contrast but inside the bandwidth the resultant optical function will be a $\pi$-phase step. Thus we have created here a spectrally exclusive phase mask with a bandwidth of $1 \mu \mathrm{m}$.

Another really interesting property of the MIM antennas is that they have a cross section of interaction much larger than their geometrical dimensions [22] (of the order of $\frac{\lambda_{\text {res }}^{2}}{2}, \lambda_{\text {res }}$ being the resonance wavelength of the antenna) allowing them to be spatially combined [23-25], which means that we can use more than one antenna in one period. Thus if we put within the same period two antennas of different lengths, the two will act independently and their individual behavior will not be modified. To achieve this, the two antennas must not interact with each other, which can be obtained with sufficiently separated resonance wavelengths which in our case is done by using sufficiently different length of gold rods.

We therefore have two exploitable properties of MIM antennas. First, in a given spectral domain we can inscribe a constant phase difference, except for diffraction, allowing realization of an exclusive phase mask, present only in a restricted spectral domain. Second, in disjoint spectral domains the phase masks produced are independent, making it possible, with a spatial co-localization of the antennas, to achieve a spectral co-localization of the phase masks. With the two previously described features of MIM antennas in mind we realize the following sample sketched in figure 2(b) to achieve spectrally exclusive phase masks. This sample is a $10 \mathrm{~mm}$ side square separated in four quadrants, each of which is composed of a periodic pattern of two antennas: one designed to work near 3 microns, the other near 5 microns. It was subcontracted to NIL Technology ApS. It consists of a silicon substrate with metallic deposition (tolerances \pm $10 \%$ ) of $5 \mathrm{~nm} \mathrm{Cr}, 100 \mathrm{~nm} \mathrm{Au}, 5 \mathrm{~nm} \mathrm{Cr}$. PECVD (tolerances $\pm 15 \%$ ) of $300 \mathrm{~nm} \mathrm{SiO}_{2}$, E-beam with positive resist, and lift off (tolerances $\pm 10 \%$ ) of $100 \mathrm{~nm}$ of gold with $5 \mathrm{~nm} \mathrm{Cr}$ as adhesive layer. (see experimental measurements in supplemental material)

To design this component, we chose three colors of interests, centered around $\lambda_{1}=3 \mu \mathrm{m}$, $\lambda_{2}=4 \mu \mathrm{m}$ and $\lambda_{3}=5 \mu \mathrm{m}$. At $\lambda_{1}$ and $\lambda_{3}$ we create two independent spectraly exclusive phase masks, respectively a horizontal $\pi$ phase step and a vertical $\pi$ phase step. By correctly choosing the dimensions we can ensure that at $\lambda_{2}$ there is not any phase contrast. With that design, the function at $\lambda_{1}$ and the one at $\lambda_{3}$ will be independent. This behavior is illustrated on figure 2(b).

The unit cell of the structure used here is illustrated in figure 2(a), to co-localize we added one antenna per period compared to the figure 1(a), so we use all the sample surface for both 


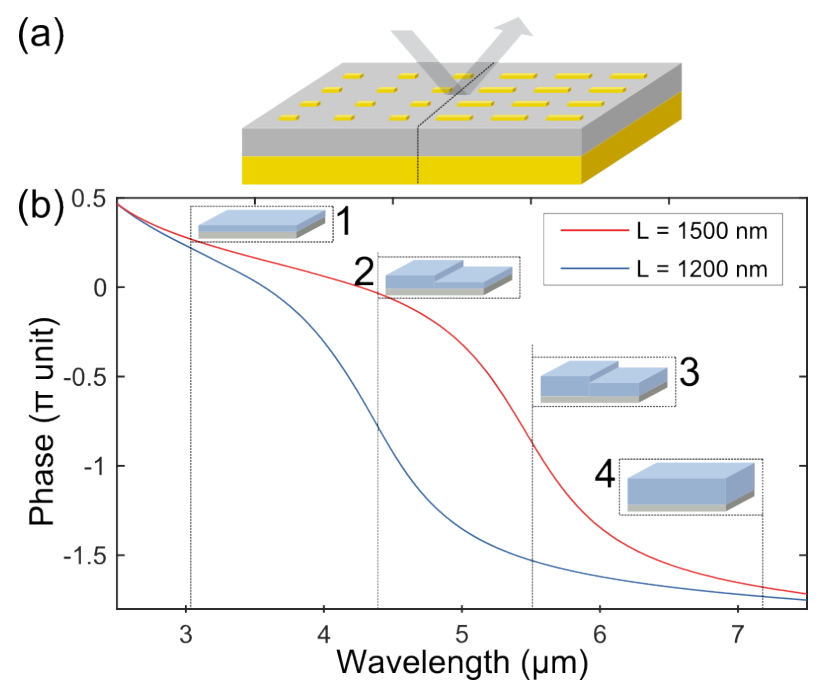

Fig. 1. (a) Two adjacent metal-insulator-metal arrays. The bottom metallic layer and the insulator layer are both continuous, while the upper gold layer is patterned with a rectangular shape. Both arrays have the same period, but different length of the metallic antenna, which generates a phase difference. (b) Phase reflected by a periodic array of MIM antennas as a function of the incident wavelength between 2.5 and $7.5 \mu \mathrm{m}$ for antenna lengths of $1200 \mathrm{~nm}$ and $1500 \mathrm{~nm}$. In insets 1-4 we highlighted different interesting behaviors of the previously described sample composed of these two antennas by showing the equivalent phase step realized by engraving a material. Before $3 \mu \mathrm{m}$ and after $7 \mu \mathrm{m}$ we have a homogeneous surface, but around $5 \mu \mathrm{m}$ we have a near $\pi$ phase step, a spectrally exclusive phase mask. Computations are led by solving Maxwell's equations thanks to the rigorous coupled wave analysis method and usual parameters.

antennas. The dimensions were chosen for use in the mid-infrared range, the following results can be transferred to other spectral domains by changing the dimensions.

The phase mask functions performed by our sample can be seen at the Point Spread Function level which is the final product of wavefront coding. Indeed a horizontal (resp. vertical) $\pi$ phase step duplicates a point source horizontally (resp. vertically) and 4 quadrants with no significant phase delay won't change the PSF.

To have a better understanding of the created function we plot in figure 3(a) the evolution of the phase for the four quadrants of the device as a function of the wavelength. We clearly observe that at the start and at the end of the graphic the four phase functions meet together so we have no phase contrast on our sample. The figure 3(b) represents phase differences between right and left quadrants (blue curves) and between upper and lower quadrants (red curves). We can quantify the spectral bandwidths of our two spectrally exclusive phase masks near $\lambda_{1}$ and $\lambda_{3}$, which are defined by $|\Delta \phi-\pi|<0.3 \pi$ and corresponds to the wavelengths were we will observe our function. They are represented as $\delta \lambda_{1}$ and $\delta \lambda_{3}$, and the first bandwidth is two times smaller than the second. Near $\lambda_{2}$ the four phase functions exhibit no sufficient phase delay so we can consider that the device will have no effect on the point spread function of our system on a bandwidth of $\Delta \lambda$. If we investigate this function at the PSF scale, before $2.5 \mu \mathrm{m}$ we observe only the point source, around $3 \mu \mathrm{m}$ we have two horizontal spots, which became one again near $4 \mu \mathrm{m}$. Around $5 \mu \mathrm{m}$ this point source duplicates again but vertically this time, and after $7 \mu \mathrm{m}$ we have the point source again.

To measure the modification of the PSF by our system, we use a collimated polarized beam 


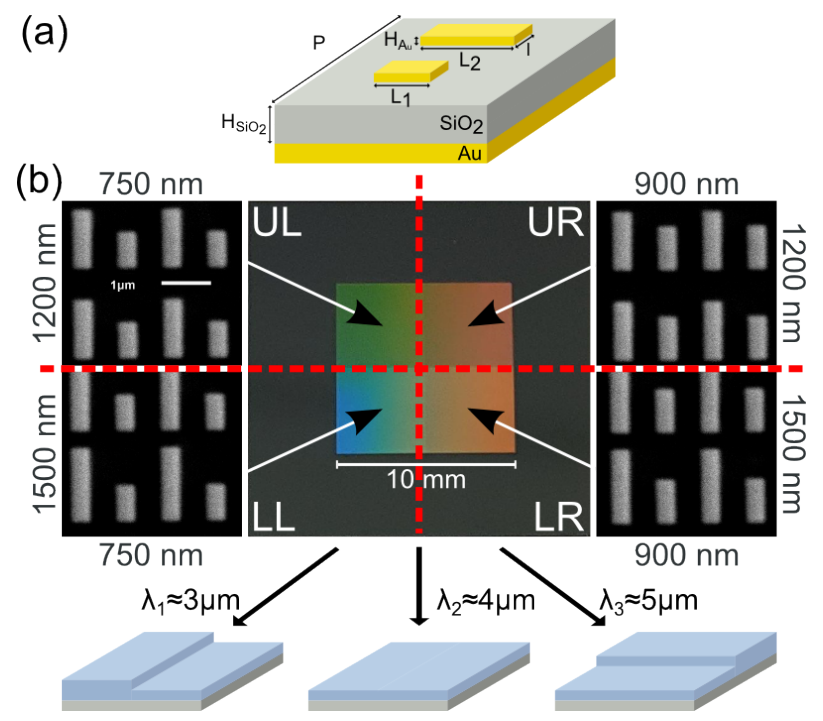

Fig. 2. (a) Representation of the unit cell used for the fabrication of the sample. The unit cell contains two antennas of different length to obtain two wavelengths of interest. The dimensions are as follow: height of insulator $H_{\mathrm{SiO}_{2}}=300 \mathrm{~nm}$, height of patterned gold layer $H_{A u}=100 \mathrm{~nm}$, period $\mathrm{P}=1800 \mathrm{~nm}$, width of the rod l $=400 \mathrm{~nm}$. The length of antennas, $L_{1}$ and $L_{2}$ will vary. (b) Image of the sample, SEM images of the periods of the different areas of interest are represented. The different lengths of the rods are remembered for each row and column. Designed feature of the sample is illustrated at three different wavelengths $\left(\lambda_{1}<\lambda_{2}<\lambda_{3}\right)$. At $\lambda_{1}$ the sample works as an horizontal phase step, exhibits no significant phase delay at $\lambda_{2}$ and works as a vertical phase step at $\lambda_{3}$.

coming from a source point, then image this source point with a cooled infrared $\mathrm{HgCdTe}$ camera after a reflection on our nano-structured sample placed in the pupil plane of the optical system represented on the schema on figure 4(a). A selection of spectral filters then allow us to observe it at the wavelengths of interest.

To have a better signal-to-noise ratio and to continuously characterize the spectral PSF of our system, we used a collimated beam coming from a Fourier Transform InfraRed spectrometer (FTIR), Bruker Vertex 70v illuminating our sample. An infrared camera (HgCdTe camera $15 \mu \mathrm{m}$ pixel pitch, working in the $3-5 \mu \mathrm{m}$ band) is used to image the source pinhole with an infrared objective. A polarizer is added at the exit of the FTIR, so that only the transverse electric or magnetic polarization is studied. We now can observe its spectral deformation by using a step-scan FTIR measurement [26] i.e. for each path difference in the FTIR interferometer the PSF is successively recorded with the infrared camera. With a spectral Fourier transform applied on each pixel we retrieve the spectral PSF.

We then compare these measurements with theoretical simulations in figure 4(b). Under TE polarized light our sample acts as a mirror with no influence on the optical PSF. Under TM polarization, around 3 microns we observe two horizontal spots, around 4 microns only one spot and at 5 microns we observe two vertical spots. So we get two independent exclusive phase masks with a return to the focal spot at 4 microns. Our bandwidth criteria $|\Delta \phi-\pi|<0.3 \pi$ corresponds in term of PSF to a relative maximal intensity in the two spot above $35 \%$.

As explained before, an interesting feature of our system is that the PSF at 3 microns is not correlated to the PSF at 4 or 5 microns. Indeed the two signatures introduced by the two spectrally phase masks are exclusive. Choosing several small antennas for the first function at 3 microns 

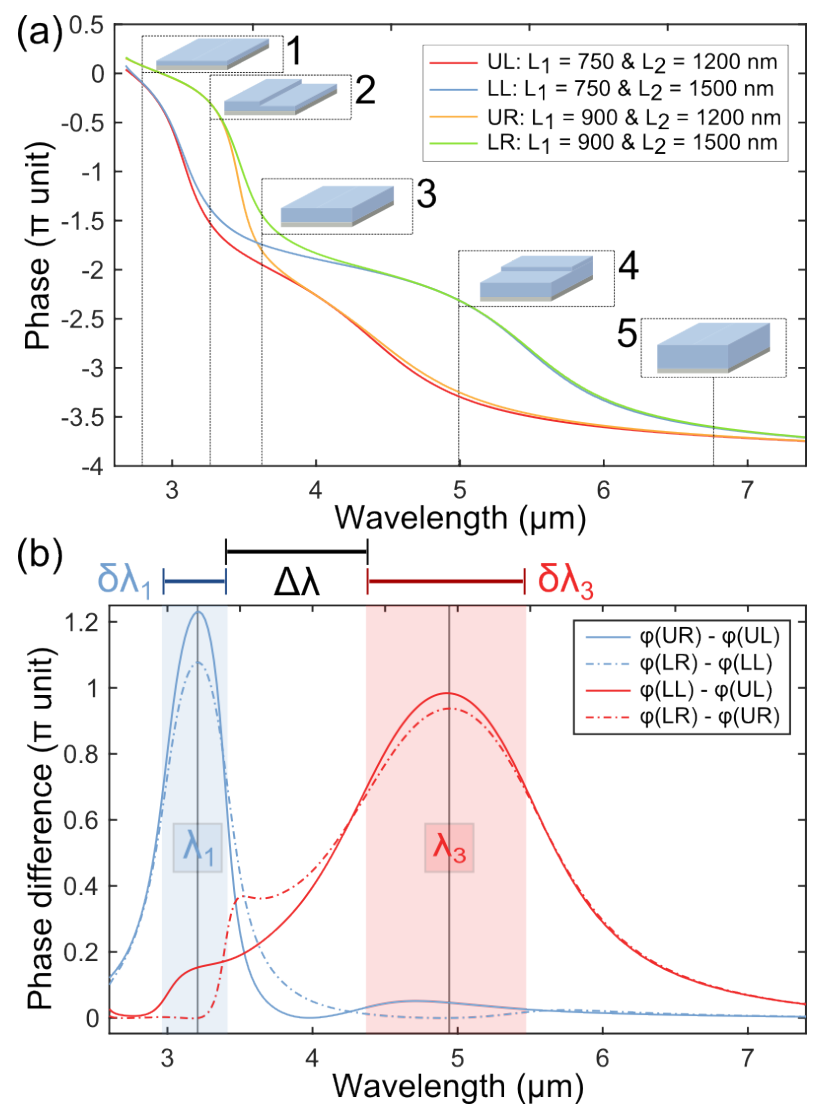

Fig. 3. (a) Phase variation of the four quadrants described on figure 2 as a function of the incident wavelength in TM polarization. In insets 1-5 we have illustrated several behaviors of the device corresponding to the characteristic phase jumps. (b) Phase differences between left and right quadrants (blue), and between upper and lower quadrants (red) as a function of the incident wavelength in TM polarization. The design wavelengths $\lambda_{1}$ and $\lambda_{2}$ and the resulting spectral bandwidths where we will observe the function $\delta \lambda_{1}$ and $\delta \lambda_{2}$ are highlighted. Between these two bandwidths our sample retrieves a neutral behavior on $\Delta \lambda$

has no significant consequences on the choice of the antennas for the function at 5 microns, we have total design freedom for both phase masks. The bandwidth of stability of the different phase steps is also another remarkable feature of our design. We have a bandwidth of more than $1 \mu \mathrm{m}$ at $5 \mu \mathrm{m}$ and of $400 \mathrm{~nm}$ at $3.2 \mu \mathrm{m}$

The use of rectangular MIM makes our system polarization-dependent. This can be avoided by using square or cross antennas. In our design, the width of $400 \mathrm{~nm}$ of antennas has no effect in the wavelengths of interest in the TE polarization (see supplemental material), so we can use the TE polarization as another degree of freedom to add more independent spectrally exclusive phase masks in our device [27].

For the demonstration sake, we co-localized two phase masks, but more complex functions can be designed. Looking at the 3 microns function in figure 4 we have a bandwidth smaller than $400 \mathrm{~nm}$, which means that while imposing a flat phase we can use at least 4 groups of antennas to perform 4 functions between 3 and 5 microns. We could say that the limit will come from the free area available inside a period of our device. Indeed a 1200x400 nm antenna takes $12 \%$ of 


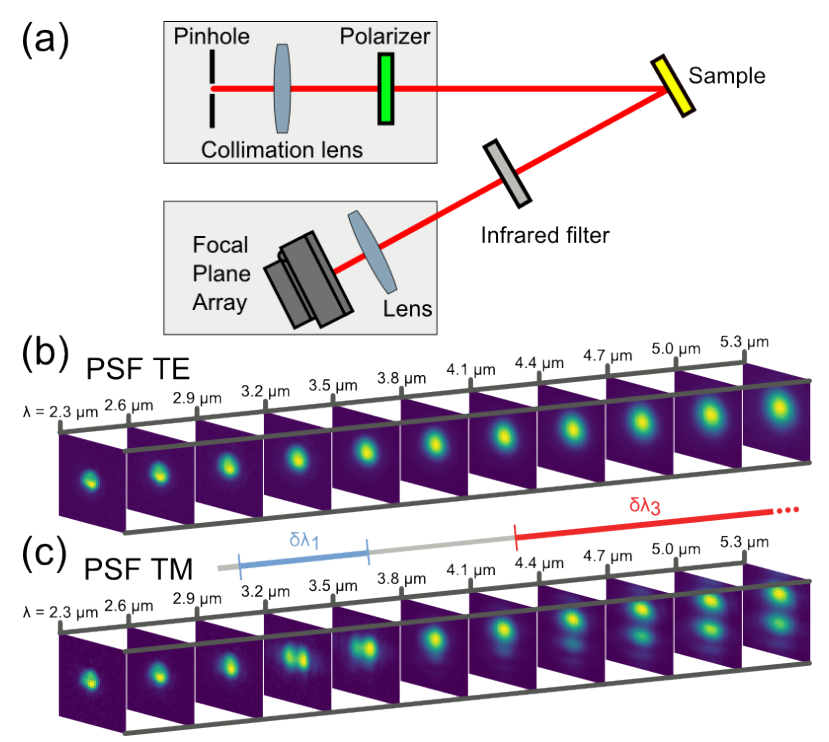

Fig. 4. (a) Experimental setup for the measurement of the spectral PSF of our system. Experimental PSF in (b) TE and (c) TM polarization at several wavelengths between 2.3 and 5.3 microns. In TE polarization our device behaves like a gold mirror. In TM polarization our device acts. Around $\lambda_{1}$ (resp. $\lambda_{3}$ ) on $\delta \lambda_{1}$ (resp. $\delta \lambda_{3}$ ) we measure two horizontal (resp. vertical) spots. Near $\lambda_{2}$ the two spots became one again. At 4.1 microns the second lobe appears, its intensity is $28 \%$ of the main one.

the surface of a $1800 \mathrm{~nm}$ square period. However, a $1200 \mathrm{~nm}$ antenna resonates at 4.3 microns, so we could increase the period of our structure to 3 microns retaining full interaction with the incident light. In this case the antenna would take only $5 \%$ of the space. Increasing the thickness of dielectric would allow to reduce the width of the antenna without losing reflectivity [21]. More complex designs have also been demonstrated by overlaying several layers of antennas [28]. Another degree of freedom come from the use of the geometric phase which permit to obtain $2 \pi$ phase shift by rotating the antenna. [29,30] We can therefore consider putting up to 4 antennas per polarization in each period.

The apparent simplicity of the duplication of source point presented in this article is not a limit, we can reproduce more complex patterns with only two levels of phase. In addition, the spatial resolution is really high since we code the phase on the scale of the period which is close to the wavelength of light.

In conclusion we have presented a new way to customize the PSF at will by using MIM antennas. These antennas allow a PSF engineering retaining complete design freedom by spectral band. We showed a simple demonstration of the independence of designs both in shape and support. Our design was simple but this technology permit more complex shapes using all the pupil independently. The choice of one phase mask does not constrain the others. To our knowledge this is the first case of engineering PSF on independent and exclusive spectral bandwidths which is particularly appealing in the context of wavefront coding to extract information in an infrared scene.

\section{Acknowledgments}

This work was supported by a DGA-AID scholarship. 


\section{Disclosures}

The authors declare that there are no conflicts of interest related to this article.

\section{References}

1. E. R. Dowski and W. T. Cathey, "Extended depth of field through wave-front coding," Appl. Opt. 34, 1859-1866 (1995).

2. S. McEldowney, "Use of wavefront coding to create a depth image," (2013). US Patent 8,558,873.

3. J. Sung, G.-Y. Lee, and B. Lee, "Progresses in the practical metasurface for holography and lens," Nanophotonics 8 , 1701-1718 (2019).

4. P. Lalanne and P. Chavel, "Metalenses at visible wavelengths: past, present, perspectives," Laser \& Photonics Rev. 11, 1600295 (2017).

5. P. Genevet, F. Capasso, F. Aieta, M. Khorasaninejad, and R. Devlin, "Recent advances in planar optics: from plasmonic to dielectric metasurfaces," Optica 4, 139-152 (2017).

6. V.-C. Su, C. H. Chu, G. Sun, and D. P. Tsai, "Advances in optical metasurfaces: fabrication and applications," Opt. Express 26, 13148-13182 (2018).

7. F. Ding, A. Pors, and S. I. Bozhevolnyi, "Gradient metasurfaces: a review of fundamentals and applications," Reports on Prog. Phys. 81, 026401 (2017).

8. S. M. Kamali, E. Arbabi, A. Arbabi, and A. Faraon, "A review of dielectric optical metasurfaces for wavefront control," Nanophotonics 7, 1041-1068 (2018).

9. P. Cheben, R. Halir, J. H. Schmid, H. A. Atwater, and D. R. Smith, "Subwavelength integrated photonics," Nature 560, 565-572 (2018).

10. Z. Yang, Z. Wang, Y. Wang, X. Feng, M. Zhao, Z. Wan, L. Zhu, J. Liu, Y. Huang, J. Xia et al., "Generalized hartmann-shack array of dielectric metalens sub-arrays for polarimetric beam profiling," Nat. Commun. 9, 1-7 (2018).

11. E. Arbabi, A. Arbabi, S. M. Kamali, Y. Horie, and A. Faraon, "Multiwavelength polarization-insensitive lenses based on dielectric metasurfaces with meta-molecules," Optica 3, 628-633 (2016).

12. E. Arbabi, A. Arbabi, S. M. Kamali, Y. Horie, and A. Faraon, "Multiwavelength metasurfaces through spatial multiplexing," Sci. Reports 6, 32803 (2016).

13. Y. Zhou, I. I. Kravchenko, H. Wang, J. R. Nolen, G. Gu, and J. Valentine, "Multilayer noninteracting dielectric metasurfaces for multiwavelength metaoptics," Nano Lett. 18, 7529-7537 (2018).

14. G. Yoon, J. Kim, J. Mun, D. Lee, K. T. Nam, and J. Rho, "Wavelength-decoupled geometric metasurfaces by arbitrary dispersion control," Commun. Phys. 2, 1-7 (2019).

15. N. Yu, P. Genevet, M. A. Kats, F. Aieta, J.-P. Tetienne, F. Capasso, and Z. Gaburro, "Light propagation with phase discontinuities: generalized laws of reflection and refraction," Science 334, 333-337 (2011).

16. Y.-W. Huang, W. T. Chen, W.-Y. Tsai, P. C. Wu, C.-M. Wang, G. Sun, and D. P. Tsai, "Aluminum plasmonic multicolor meta-hologram," Nano Lett. 15, 3122-3127 (2015).

17. P. Bouchon, C. Koechlin, F. Pardo, R. Haïdar, and J.-L. Pelouard, "Wideband omnidirectional infrared absorber with a patchwork of plasmonic nanoantennas," Opt. letters 37, 1038-1040 (2012).

18. Q. Lévesque, M. Makhsiyan, P. Bouchon, F. Pardo, J. Jaeck, N. Bardou, C. Dupuis, R. Haïdar, and J.-L. Pelouard, "Plasmonic planar antenna for wideband and efficient linear polarization conversion," Appl. Phys. Lett. 104, 111105 (2014).

19. S. Zhang, M.-H. Kim, F. Aieta, A. She, T. Mansuripur, I. Gabay, M. Khorasaninejad, D. Rousso, X. Wang, M. Troccoli, N. Yu, and F. Capasso, "High efficiency near diffraction-limited mid-infrared flat lenses based on metasurface reflectarrays," Opt. Express 24, 18024-18034 (2016).

20. A. Arbabi, Y. Horie, A. J. Ball, M. Bagheri, and A. Faraon, "Subwavelength-thick lenses with high numerical apertures and large efficiency based on high-contrast transmitarrays," Nat. Commun. 6, 1-6 (2015).

21. A. Pors and S. I. Bozhevolnyi, "Plasmonic metasurfaces for efficient phase control in reflection," Opt. Express 21, 27438-27451 (2013).

22. P. Chevalier, P. Bouchon, J. Jaeck, D. Lauwick, N. Bardou, A. Kattnig, F. Pardo, and R. Haïdar, "Absorbing metasurface created by diffractionless disordered arrays of nanoantennas," Appl. Phys. Lett. 107, 251108 (2015).

23. M. Makhsiyan, P. Bouchon, J. Jaeck, J.-L. Pelouard, and R. Haïdar, "Shaping the spatial and spectral emissivity at the diffraction limit," Appl. Phys. Lett. 107, 251103 (2015).

24. D. Wen, F. Yue, G. Li, G. Zheng, K. Chan, S. Chen, M. Chen, K. F. Li, P. W. H. Wong, K. W. Cheah et al., "Helicity multiplexed broadband metasurface holograms," Nat. Commun. 6, 1-7 (2015).

25. Q. Fan, P. Huo, D. Wang, Y. Liang, F. Yan, and T. Xu, "Visible light focusing flat lenses based on hybrid dielectric-metal metasurface reflector-arrays," Sci. Reports 7, 1-9 (2017).

26. S. Rommeluère, R. Haïdar, N. Guérineau, J. Deschamps, E. De Borniol, A. Million, J. Chamonal, and G. Destefanis, "Single-scan extraction of two-dimensional parameters of infrared focal plane arrays utilizing a fourier-transform spectrometer," Appl. Opt. 46, 1379-1384 (2007).

27. J. B. Mueller, N. A. Rubin, R. C. Devlin, B. Groever, and F. Capasso, "Metasurface polarization optics: independent phase control of arbitrary orthogonal states of polarization," Phys. Rev. Lett. 118, 113901 (2017). 
28. M. M. Hossain, B. Jia, and M. Gu, "A metamaterial emitter for highly efficient radiative cooling," Adv. Opt. Mater. 3, 1047-1051 (2015).

29. G. Zheng, H. Mühlenbernd, M. Kenney, G. Li, T. Zentgraf, and S. Zhang, "Metasurface holograms reaching $80 \%$ efficiency," Nat. Nanotechnol. 10, 308-312 (2015).

30. M. Kim, A. M. Wong, and G. V. Eleftheriades, "Optical huygens' metasurfaces with independent control of the magnitude and phase of the local reflection coefficients," Phys. Rev. X 4, 041042 (2014). 